This is an Accepted Manuscript of an article published by Sanna Järvelä \& Maria Bannert, (2019) Temporal and adaptive processes of regulated learning : What can multimodal data tell?, Learning and Instruction, 101268, available online: https://doi.org/10.1016/j.learninstruc.2019.101268 


\section{Temporal and adaptive processes of regulated learning - what can multimodal data tell?}

The aim of the special issue is to present studies and discuss about how temporal and adaptive processes or regulation of learning can be understood by using multimodal data. In self-regulated learning research (SRL) progress has been made evidencing that self-, co- and socially shared regulation of learning is critical for learning success (e.g. Hadwin, Järvelä, \& Miller, 2018; Molenaar, \& Järvelä, 2014). More is also understood with regard to the role of regulatory processes in today's social, interactive, and technology enriched learning situations with an increased evidence how adaptive learning technologies (ALT) can be used for supporting critical processes in students' regulation (Azevedo \& Gasevic, 2019). For example, computer supported collaborative learning data, such as collaborative and socioemotional interactions, transactions and knowledge building processes have temporal (in terms of time) and multidimensional (in terms of interactions in affect and cognition) nature, which in the optimal cases of collaboration are successfully shared and monitored in between the collaborating partners (Kirschner et al., 2018). Another example is metacognitive scaffolding in ALTs, which is supposed to support the learner's regulation processes and is examined by looking at the temporal order of logfile and think aloud data (Bannert et al., 2015). Moreover, metacognitive tutoring with ALT is based on the assumption of the temporal character of student's regulation processes which is considered in form of analyzing the sequential structure in a learning process by means of logfile events and/or emotion recognition data.

Contemporary perspectives view SRL as a cyclical complex metacognitive and social process that involves adapting cognition and metacognition, motivation, emotion, and behavior. This is to say, regulation involves cyclical adaptation. It is neither static nor a state, but rather a series of contingencies over time (Winne, 2019). What it still not clear, is when those actions take place, how they influence each other and how they refer to learning performance. However, this information is needed for targeted support in regulation in an individual as well as in group learning setting/s. In current research, there is a lack of studies examining change of regulatory processes and types of regulation over time as a variable in research about learning regulation. This is primarily because of the limited methodological ways to capture this evolving process. The contributors of this special issue have been pioneers in the use of multimodal data in SRL research making effort on revealing and understanding the details in the complex nature of SRL as an event that unfolds over time. All of the contributors have unique methodological and analytical solutions to target temporal and adaptive processes of regulated learning. Since the studies share a strong theoretical grounding, but vary with the settings and a set of methods, the whole special issue helps to uncover still unknown processes of learning regulation.

Still, it is important to stress that humans are agentic, interpretative and reflective in combination with their tendency to make extensive use of situational resources, such as physical, symbolic and social. Reimann, Markauskaite and Bannert (2014) claim that it is highly unlikely that we could ever predict SRL behaviour with a reasonable amount of certainty by, for example, using only patterns of learning events. Learners' contextual and social interactions and their specific interpretations of the situation (Järvenoja, Järvelä \& Malmberg, 2017) make "errors" for patterns and causalities. Hence, the studies in this special issue have looked at both causalities and patterns and mechanisms of contextual and social interaction. The authors use multiple channel data to cover many different processes of SRL and to investigate the interplay of all these different processes.

In this special issue Järvelä et al. give an overview of important research questions needed to be solved and demonstrate how they can be addressed by triangulating multiple sources of data modalities. Their study uses multimodal data triangulation to study SRL processes during authentic 
collaborative learning. They match process data based on the time-stamped information related to each data source and video data coding provides the grounding for contextualizing data and triangulation. They demonstrate how by data triangulating it is possible to study a number of features of regulated learning in collaboration, such as how affective responses to situations may interact with cognitive strategic actions in the learning situation, the temporal and cyclical nature of regulatory processes, and the occurrence of different types of regulation during collaborative learning.

Lajoie et al. study affect in SRL using multimodal data in the context of a clinical reasoning task for medical students. They examine dynamic co-occurrences of SRL processes with emotions and motivation in the ALT by using sequential data mining techniques. They explored the temporal nature of cognition, affect, motivation, and self-regulation by data collected on self-regulated learning processes through think-aloud analyses; emotion data through facial expressions; and achievement goal orientations and habitual emotion regulation strategies through self-report questionnaires. Their results from sequential data mining techniques and quantitative analyses suggest differences in highand low performing medical students affective, cognitive and SRL processes and transitions in between them.

Taub et al. focus on student's note taking and summarizing while studying with ALT and analyse temporal dynamics of monitoring and regulation of learning by using multichannel data. They studied students' evidence scores of emotions while they engaged in cognitive and metacognitive selfregulated learning processes as they learned about the circulatory system with a hypermedia-based intelligent tutoring system. They detected students' cognitive and metacognitive processes and examined how the computed scores related to mean evidence scores of emotions and overall learning. Their results indicated positive and negative predictions in between emotions and cognition and increase understanding the beneficial role of negative emotions during learning with advanced learning technologies.

Engelmann and Bannert examine the temporal structure of student's SRL-processes induced by prompts using educational process mining techniques. In their experiment students received metacognitive prompts or no prompts during learning in a hypermedia learning environment while thinking aloud. Student's verbal protocols were coded post-hoc in respect to different self-regulation events and the coded data were used for process mining to investigate the learning processes more deeply. Contrary to prior research no positive prompting effects were found in respect to learning performance. An in-depth analysis highlights the potential of process mining techniques for educational research by demonstrating that the process models for both treatment groups (prompted vs. not prompted group) are - contrary to expectations - very similar, which may explain the lack of performance differences.

Lim et al. investigate the impact of a learning analytics-based feedback system on students' SRL and academic achievement in a large, first-year undergraduate course. Student's learning operations are analysed by using log data from the learning management system and e-book and linked with performance on course assessments. By use of machine learning and common statistical methods the patterns of online self-regulated learning operations over the course of the semester were identified. Results show, that students supported by the peronalized feedback showed not only significantly different patterns in SRL but also better final grades compared to students of the un-supported control group.

Greene et al. study characteristics of students' self-regulation during science learning spanning inclass and out-of-class events in the learning management system along with the other process data to understand how they contingently precede and predict one another, and further predict students' 
achievement. They applied multimodal online interaction trace data to investigate the temporal nature of self-regulation during science education. Their latent profile analyses pointed out differences in self-regulatory processing and gave implications for identifying and supporting students' active science learning.

Finally, Molenaar et al. study ALT trace data when learning with tablets to get deeper insights into students' SRL by using the method of moment-by-moment learning curves. In particular, they examined how learners regulate their effort, accuracy and learning with adaptive learning technologies and found large differences in these variables. Moreover, their results indicate that moment-by-moment learning curves seem to be valuable indicators of accuracy and learning and, hence, provide deeper insight into how students regulate their accuracy and learning over time while learning with ALTs. They suggest that moment-by-moment learning curves could potentially be used in interventions to support student's SRL by personalized visualizations or to support teachers by presenting such curves in digital diagnostic tools.

In their efforts for understanding temporal and adaptive process of regulated learning, the research teams of this special issue have mostly worked in multidisciplinary teams. They have productively bridged educational psychology to data sciences and imported statistical methods from other disciplines in their attempts to create theoretical explanations to their research questions. Challenges have emerged and they have been discussed in the papers by each team. The contributions do not necessarily meet the common standards of empirical work in the field of learning and instruction. They lie between educational science and the new field of data sciences/learning analytics, and, thus, requires different procedures and standards which are currently developed. Nevertheless, the special issue also provokes the field to continue discussion.

We thank all reviewers for their constructive feedback and valuable comments on the present manuscripts. Moreover, we especially thank the two experts in the field - Allyson Hadwin and Peter Reimann - who critically conclude how the methods used and the empirical findings received can advance the field by progressing the theory and development of adaptive support for regulation of learning. Their insights and recommendations will push the current research teams work and the field in general forward. 